\title{
802GHz INTEGRATED HORN ANTENNAS IMAGING ARRAY
}

\author{
Walid Y. Ali-Ahmad, ${ }^{1}$ Gabriel M. Rebeiz, ${ }^{1}$ \\ Hemant Davé, ${ }^{2}$ and Gordon Chin ${ }^{2}$ \\ ${ }^{1} N A S A / C e n t e r$ for Space Terahertz Technology \\ Electrical Engineering and Computer Science Department \\ University of Michigan \\ Ann Arbor, $M 1$ 48109-2122 \\ ${ }^{2}$ Planetary Systems Branch \\ NASA/Goddard Space Flight Center \\ Greenbelt, MD 20771
}

Received March 7, 1991

\begin{abstract}
This short paper presents the pattern measurements at $802 \mathrm{GHz}$ of a single element in a 256-element integrated horn imaging array. The integrated-horn antenna consists of a dipole-antenna suspended on a $1 \mu \mathrm{m}$ dielectric membrane inside a pyramidal cavity etched in Silicon. The theoretical far-field patterns, calculated using reciprocity and Floquet-modes representation of the free-space field, agree well with the measured far-field patterns at $802 \mathrm{GHz}$. The associated directivity for a $1.40 \lambda$ horn aperture, calculated from the measured $\mathrm{E}$ and $\mathrm{H}$-plane patterns is $12.3 \mathrm{~dB} \pm 0.2 \mathrm{~dB}$. This work demonstrates that high-efficiency integrated-horn antennas are easily scalable to terahertz frequencies and could be used for radio-astronomical and plasma-diagnostic applications.
\end{abstract}




\section{INTRODUCTION}

The integrated horn antennas array has been successfully demonstrated at $94 \mathrm{GHz}$ and $240 \mathrm{GHz}$ [1-4]. The antenna array is fully monolithic, free of dielectric and surface-wave losses and easily reproducible for array applications. The horn antennas are typically between $1 \lambda$ and $1.5 \lambda$ square, and show excellent patterns at $93 \mathrm{GHz}$ with a directivity around $10-13 \mathrm{~dB}$. The integrated-horn antenna has a measured aperture efficiency of $72 \%$ at $93 \mathrm{GHz}$ [2], and the design can be easily extended to submillimeter-wave applications. The horn array is composed of two stacked Silicon wafers with a $<100\rangle$ crystal orientation. The opening of the front wafer determines the aperture size, while its thickness determines the position of the dipole feed inside the horn. The horn cavity is made by anisotropic etching of the Silicon wafers. The etching process forms pyramidal holes bounded by the $\langle 111\rangle$ crystal planes, and produces a horn flare-angle of $70.6^{\circ}$ [5]. The dipole antennas and detectors are fabricated on membranes which are formed by depositing a 3-layer $\mathrm{SiO}_{2} / \mathrm{Si}_{3} \mathrm{~N}_{4}$ dielectric on the front wafer, and etching the underlying Silicon until the transparent membrane appears. The two wafers are finally aligned and assembled together to form the horn antenna array (Fig. 1).

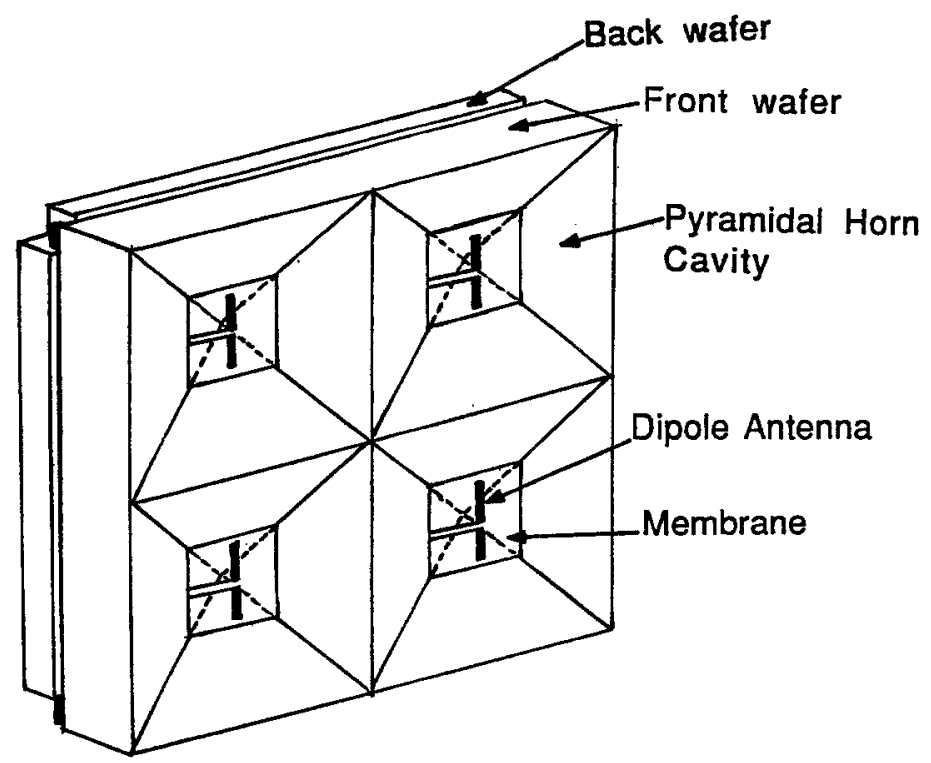

Figure 1: An integrated horn-antennas array with single polarization. 


\section{DESIGN AND MEASUREMENTS}

The theoretical far-field patterns of a horn antenna in a two-dimensional array is calculated by approximating the horn structure with a cascade of rectangular waveguide sections. The fields in each waveguide section are given by a linear combination of TE/TM waveguide modes and the fields in space are given by a discrete spectrum of Floquet modes. The boundary conditions are matched at each of the waveguide sections and at the aperture of the horn. Reciprocity is then used to relate the fields of a dipole to the dipole in the horn cavity. The reader is referred to [1] for a complete description of the theory. A microwave scale model of the horn antenna was built at $1.1 \mathrm{GHz}$ and impedance measurements were done for a $1.40 \lambda$ square horn. The resonant feed-dipole impedance can be varied between $30 \Omega$ and $80 \Omega$ for feed positions between $0.3 \lambda$ and $0.5 \lambda$ from the apex of the horn and this impedance range is quite suitable for Schottky-diode and SIS receivers [4]. For a feed position of $0.38 \lambda$, The resonant length of the feed dipole is $0.4 \lambda$ and the resulting bandwidth is about $10 \%$ (Fig. 2). An array of $16 \times 16$ integrated horn antennas was built at $802 \mathrm{GHz}$ with $1.40 \lambda$ horn opening with a feed dipole of length $0.4 \lambda$ positioned $0.38 \lambda$ from the apex (Fig. 3).

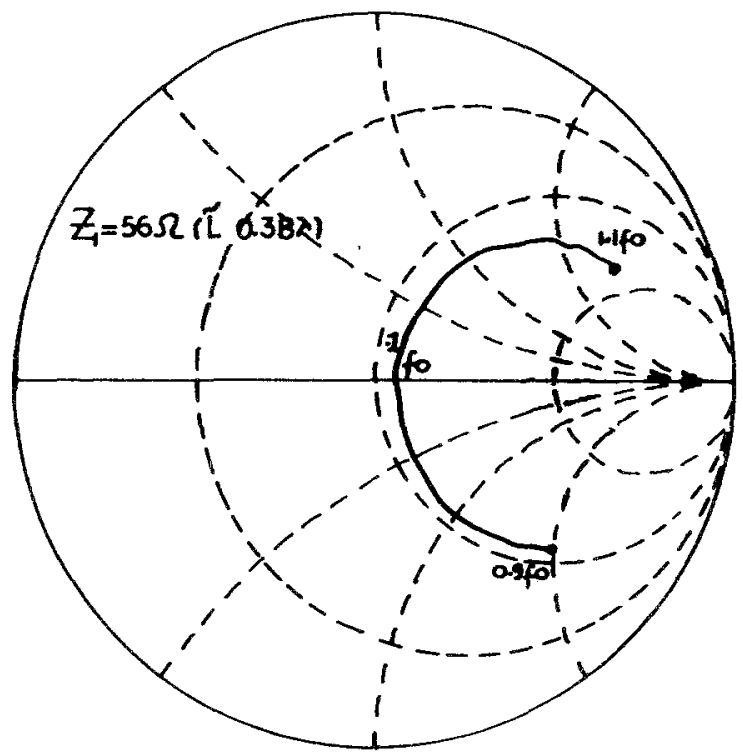

Figure 2: Resonant input impedance for a feed dipole at $0.38 \lambda$ from the apex, and for a $1.35 \lambda$ horn opening. 
The far-field patterns of a single integrated horn antenna in an array were measured using a far-infrared laser tuned at $802 \mathrm{GHz}$. The detectors are $4 \mu \mathrm{m}$-square Bismuth microbolometers and are integrated at the center of each dipole antenna. Figure 4 shows the experimental $\mathrm{E}$ and $\mathrm{H}$-plane patterns which agree well with the theoretical patterns. The $\mathrm{H}$-plane is smooth due to the $\mathrm{TE}_{10}$ tapering of the electric field across the aperture. In the case of the E-plane, the horn sees the array and the spikes and nulls in the patterns are due to specific Floquet-modes. The $\mathrm{E}$ and $\mathrm{H}$-plane $10 \mathrm{~dB}$-beamwidths are $80^{\circ}$ and $90^{\circ}$ respectively and the far-field pattern matches well an f/0.8 imaging system. The directivity of a single horn antenna calculated from the measured $\mathrm{E}$ and $\mathrm{H}$-plane patterns is $12.3 \mathrm{~dB} \pm 0.2 \mathrm{~dB}$. The pattern is quite symmetrical as is evident from the measured $45^{\circ}$-plane pattern (Fig. 5). The radiated power is well confined to a main central beam, and nearly $88 \%$ of the radiated power is within a $100^{\circ}$ beamwidth. The resulting spillover loss is around $0.5 \mathrm{~dB}$.

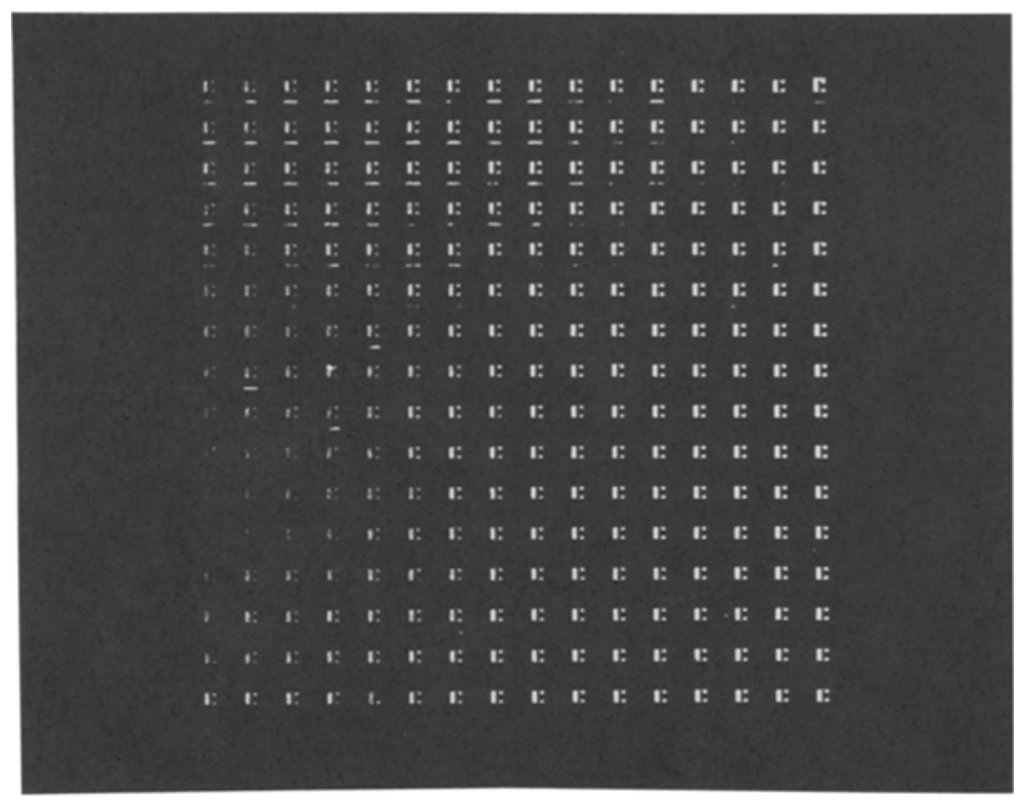

Figure 3: a $16 \times 16$ integrated horn-antennas array built at $802 \mathrm{GHz}$. 


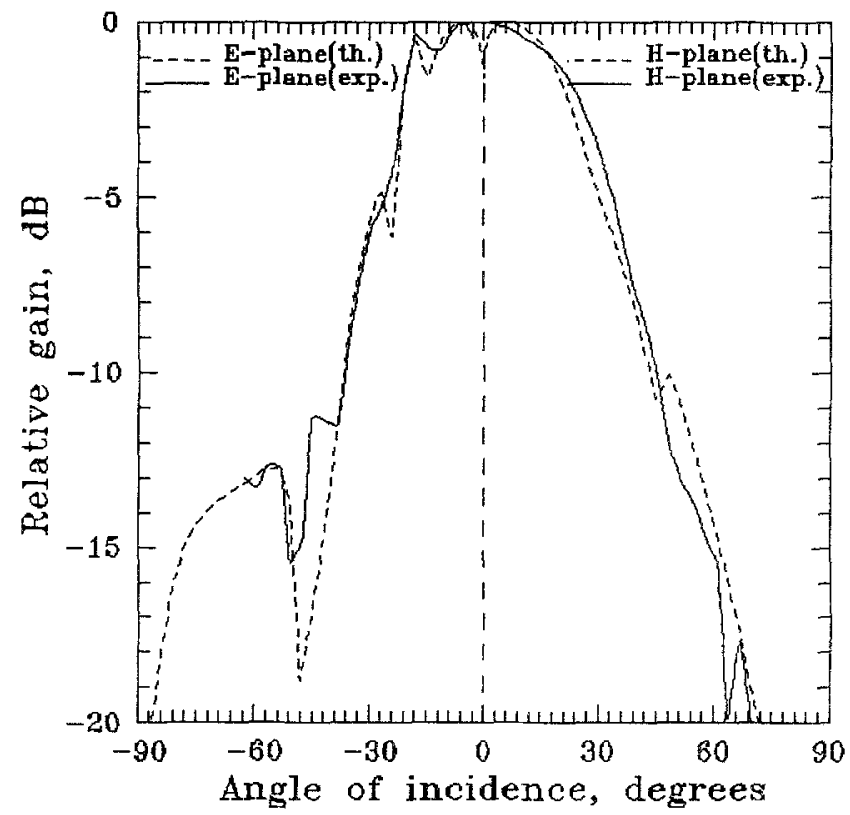

Figure 4: Theoretical and experimental E and H-plane patterns.

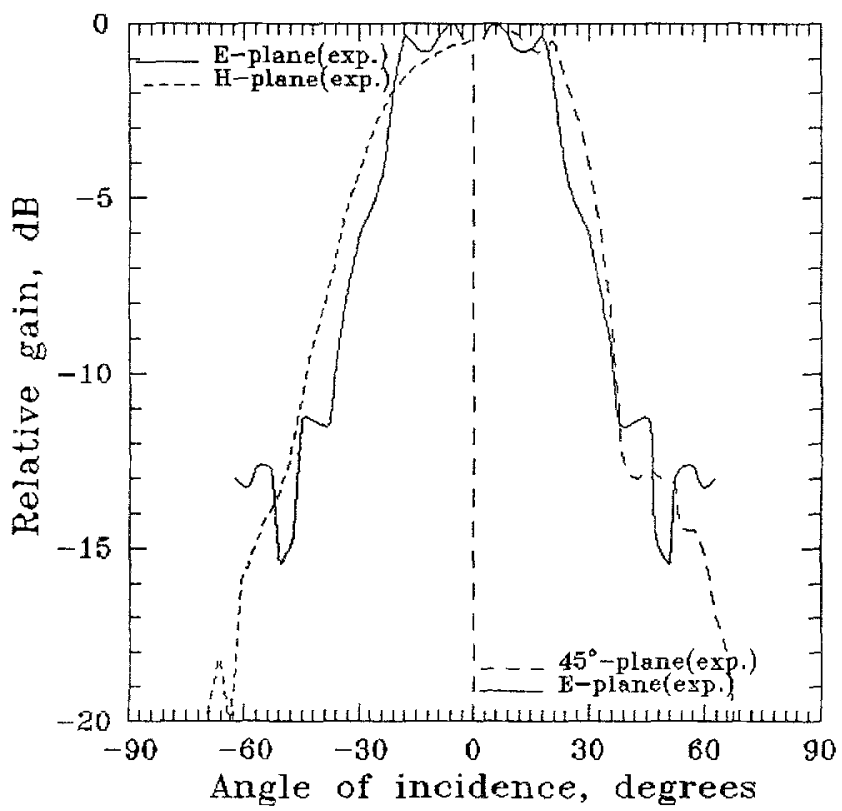

Figure 5: Experimental E, $\mathrm{H}$ and $45^{\circ}$-plane patterns. 


\section{CONCLUSIONS}

An efficient antenna has been built at $802 \mathrm{GHz}$ and is suitable for submillimeter-wave receivers. In the future, it should be also possible to integrate SIS detectors on the membranes. The available space on the back of the wafer on which the dipole antennas are integrated allows the integration of the RF, LO and IF circuitry of the receiver.

\section{ACKOWLEDGEMENTS}

This work was supported by the NASA/Center for Space Terahertz Technology at The University of Michigan, Ann Arbor.

\section{REFERENCES}

[1] G.M. Rebeiz, D.P. Kasilingan, P.A. Stimson, Y. Guo, and D.B. Rutledge, "Monolithic millimeter-wave two-dimensional horn imaging arrays," IEEE Trans. Antennas Propag., vol. Ap-28, Sept. 1990.

[2] Y. Guo, K. Lee, P. Stimson, K.A. Potter, and D.B. Rutledge, "Aperture efficiencies of chemically etched horns," IEEE/AP-S Symposium, Dallas, Texas, 1990.

[3] W.Y. Ali-Ahmad, and G.M. Rebeiz, " $92 \mathrm{GHz}$ dual-polarized integrated horn antennas," accepted for publication in the IEEE-Trans. Antennas Propag., Dec. 1990.

[4] G.V. Eleftheriades, W.Y. Ali-Ahmad, L.P. Katehi. and G.M. Rebeiz, "Millimeter-wave integrated-horn antennas, Part I: Theory," and "... , Part II: Experiment," submitted for publication in the IEEETrans. Antennas Propag., Dec. 1990.

[5] K.E. Peterson, "Silicon as a mechnaical material," Proc. of the IEEE, vol. 70, pp. 420-457, 1982. 